\title{
Dynamic Detection and Removal of Inactive Clauses in SAT with Application in Image Computation
}

\author{
Aarti Gupta \\ CCRL, NEC USA \\ 4 Independence Way \\ Princeton, NJ 08540 \\ agupta@nec-lab.com
}

\author{
Anubhav Gupta \\ School of Computer Science \\ Carnegie Mellon University \\ Pittsburgh, PA 15213 \\ anubhav@cs.cmu.edu
}

\author{
Zijiang Yang, Pranav Ashar \\ CCRL, NEC USA \\ 4 Independence Way \\ Princeton, NJ 08540 \\ jyang,ashar@nec-lab.com
}

\begin{abstract}
In this paper, we present a new technique for the efficient dynamic detection and removal of inactive clauses, i.e. clauses that do not affect the solutions of interest of a Boolean Satisfiability (SAT) problem. The algorithm is based on the extraction of gate connectivity information during generation of the Boolean formula from the circuit, and its use in the inner loop of a branch-and-bound SAT algorithm. The motivation for this optimization is to exploit the circuit structure information, which can be used to find unobservable gates at circuit outputs under dynamic conditions. It has the potential to speed up all applications of SAT in which the SAT formula is derived from a logic circuit. In particular, we find that it has considerable impact on an image computation algorithm based on SAT. We present practical results for benchmark circuits which show that the use of this optimization consistently improves the performance for reachability analysis, in some cases enabling the prototype tool to reach more states than otherwise possible.
\end{abstract}

\section{INTRODUCTION}

Checking Boolean Satisfiability (SAT) is at the core of a number of applications in VLSI CAD including combinational verification, ATPG, timing analysis, synthesis and, recently, reachability analysis and model checking. Acceleration of SAT can have significant impact in terms of improving the quality of these applications. SAT has received considerable attention in the recent past, focusing both on basic improvements in the SAT algorithms [7, 11, 14, 19], and on various applications like Automatic Test Pattern Generation (ATPG) [12], equivalence checking [5, 9], bounded model checking (BMC) [2]. Recently, combining SAT techniques with BDDs has been shown to be effective for image computation with application in state reachability analysis of sequential circuits [10].

Typical SAT solvers are based on a Davis-Putnam style branch-and-bound algorithm, and include considerable so-

Permission to make digital or hard copies of all or part of this work for personal or classroom use is granted without fee provided that copies are not made or distributed for profit or commercial advantage and that copies bear this notice and the full citation on the first page. To copy otherwise, to republish, to post on servers or to redistribute to lists, requires prior specific permission and/or a fee.

DAC 2001, June 18-22, 2001, Las Vegas, Nevada, USA

Copyright 2001 ACM 1-58113-297-2/01/0006 ...\$5.00. phistication in heuristics for decision making, implication gathering, and backtracking [15, 18, 22]. However, missing from these efforts is exploitation of the fact that most SAT problems arising in VLSI CAD are derived from logic gate netlists. Logic gate netlists have some special properties related to how gates are connected together to realize circuit functionality. These include properties like the inputto-output flow of information, limitation on the fanout and fanin of each gate, and the connectivity and signal value dependence dynamically changing the controllability and observability of a gate. To the best of our knowledge, such properties have not being exploited in current SAT packages.

On the other hand, circuit structure has been effectively utilized in many SAT applications, e.g. ATPG [1], BMC [20]. However, this utilization has been mostly application-specific. For example, justification/propagation frontiers in ATPG, and unrolled transition relations in BMC, are used for more effective decision heuristics in the associated SAT procedures.

Our motivation is to exploit circuit structure in order to improve the performance of generic SAT applications arising in VLSI CAD. In particular, we use the notion of unobservability of gates at circuit outputs to reduce the size of the SAT sub-problems. Like all search algorithms, the effectiveness of a SAT algorithm is based on the amount of pruning of the search space it enables. The amount of pruning, in turn, is directly affected by the decision making heuristics and implication methods. Typically, their effectiveness is inversely proportional to the size of the SAT problem. Thus, the size of a SAT problem affects not only the size of the search space, but also the effectiveness of pruning methods.

\subsection{Our Contributions}

Our optimization technique for reducing the size of SAT sub-problems uses the notion of unobservability for the dynamic detection and removal of inactive clauses, i.e. clauses which do not affect the SAT solutions of interest. Rather than perform the analysis on the circuit structure, our proposed optimization works directly on the CNF (Conjunctive Normal Form) description of the circuit. Since, typical SAT solvers use a CNF description of the problem, this avoids the overhead of multiple representations.

We tag the variables and clauses of a CNF description with circuit connectivity information. This is done once at the time of generating the CNF description from the circuit structure. Later, this information is used repeatedly in the inner loop of a typical Davis-Putnam style branch-and- 
bound SAT algorithm [14] to perform a backward traversal in order to remove inactive clauses. Note that the connectivity information in the CNF description is adequate for any traversal of the corresponding circuit structure. This can be potentially used in other optimizations, besides detection of inactive clauses.

Our proposed algorithms for generating the connectivity information, and its use for detection of inactive clauses are efficient. In particular, the algorithm for detecting inactive clauses involves marking all clauses and variables in the worst case. Depending upon the application, this cost can be either incurred at every decision level, or amortized over several decision levels within the SAT search. The important point to note is that our optimization provides a uniform way to exploit circuit connectivity information, and has the potential to accelerate all SAT applications in which the SAT formula is derived from a logic circuit representation. In this paper, we focus on a SAT-based image computation application for reachability analysis [10]. For this application, the overhead of detecting inactive clauses at every decision level is negligible. Our experimental results show that the use of this technique consistently improves the performance of our prototype tool, in some cases enabling it to reach more states than otherwise possible.

\subsection{Related Work}

While our technique provides a clear benefit to SAT algorithms, it is also important to put our ideas in the context of the large body of work in combinational ATPG [1]. Unlike traditional SAT, conventional ATPG algorithms operate on data structures at the level of the circuit structure itself. As a result, all circuit information, including gate connectivity, is directly available to these algorithms. Indeed, dynamic detection of inactive circuit regions is performed by the justification-propagation operations, which are at the core of ATPG algorithms. However, there is no explicit removal of these inactive regions. In particular, the overhead of propagating values through inactive regions is not avoided. In contrast, our proposed technique effectively removes the inactive regions (clauses) from further consideration. Furthermore, it provides a way to exploit the gate connectivity information within a traditional SAT formulation based on CNF formulas. Our ideas are also peripherally related to, but go beyond, the notion of dominators in ATPG. This is clarified further in Section 2.

In terms of detecting redundant clauses in a SAT formula, a recent effort [13] proposed the use of clause dominance in the inner loop of the SAT algorithm. Unfortunately, the effort required for detection of dominating clauses is high, and it typically negates the gains arising out of clause removal.

\section{INACTIVE CLAUSES}

As a motivating example, consider the circuit shown in Figure 1(a). Suppose, the signal $a$ has been set to 0 , say, within the branch-and-bound SAT search. This implies a 0 on signal $c$ at the output of the AND gate. As a result, signal $b$ becomes unobservable at the output. In addition, any gate which fans out only to the transitive fanin cone of signal $b$, i.e. is dominated by $b$, also becomes unobservable at the output. As a result, all clauses associated with such gates are inactive, in that they can be safely removed without affecting the SAT solution for the output. Note that the status of these clauses, i.e. whether or not they are inactive,

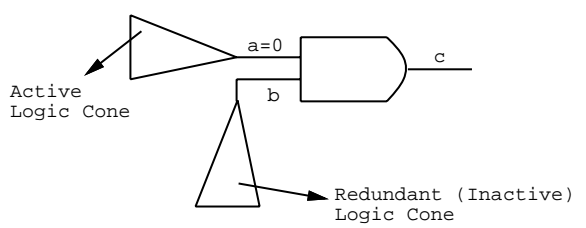

(a)

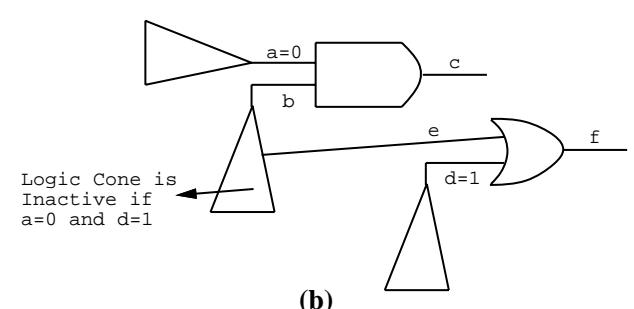

Figure 1: Circuit Example

depends on the values taken by variables $a$ and $b$. In other words, it is determined dynamically within the SAT search which assigns/implies values for these variables.

Now, consider a similar example shown in Figure 1(b). Again, let signal $a$ be set to 0 , implying a 0 on signal $c$, and making signal $b$ unobservable. Note that signal $b$ does not statically dominate all gates in its fanin cone, since the shown signal $e$ fans out to another gate. Consider the case where signal $e$ is also unobservable, due to signal $d$ being 1 , thereby implying a 1 on the output signal $f$ of that OR gate. In this case again, the clauses associated with all gates in cone of $b$ are inactive, since these gates fan out to unobservable signals only.

Our ideas are peripherally related to dominators in combinational ATPG [1]. A gate $G$ is called a structural dominator of a gate $H$ if all paths from $H$ to an output must pass through $G$. If gate $G$ is a dominator of all gates and primary inputs in its transitive fanin, the justification process [1] in ATPG can stop at $G$. It must be separately checked that both $0 / 1$ values can be generated at $G$ for some input combinations. Our ideas go beyond the way dominators are used in ATPG. First off, the notion of structural dominators in testing is purely static. On the other hand, our notion of inactive clauses is a function of dynamic values on variables, i.e. whether or not a clause is inactive changes dynamically during the SAT search. Secondly, inactive clauses can be completely removed from the sub-problem at that point in the SAT search. In contrast, ATPG algorithms use the dominators only to partition the overall problem into one for the dominated portion of the circuit, and another for the rest.

Given a gate-level circuit description, a simple backward traversal starting from circuit outputs can be performed in order to identify unobservable gates, and all clauses associated with such gates are guaranteed to be inactive. However, this requires switching back and forth between a circuit structure description and a CNF description. Since our goal is to use inactive clause detection in the inner loop of a SAT search, it is desirable to reduce this overhead. Our proposed technique is to perform the analysis on the CNF description itself. The key is to associate the connectivity information between signals and gates of the circuit structure description, with the variables and clauses of the CNF description. This 
information is generated statically at the time of generating the CNF formula from the circuit structure, and is used dynamically within the SAT search to detect and remove inactive clauses. The next two subsections describe these algorithms in detail.

\subsection{Structural Information for CNF}

Typically, the signals of a circuit are represented as variables, and the functionality of each gate is expressed as clauses for generation of a CNF formula from a circuit structure [12]. To capture the circuit structural connectivity for a backward traversal, we associate the set of clauses for each gate as the fanin list for the variable which denotes the output of that gate. Thus, each variable (except a primary input variable) is associated with a fanin list of clauses. Note also that the other clauses which a variable might appear in, e.g. clauses for gates in its fanout, are not required for backward traversal. (For a forward traversal, we could similarly associate a fanout list of clauses with each variable.)

The pseudo-code of our algorithm for generating these fanin lists along with CNF generation is shown in Figure 2. Here, we assume the availability of a gate-level circuit description in terms of simple Boolean gates.

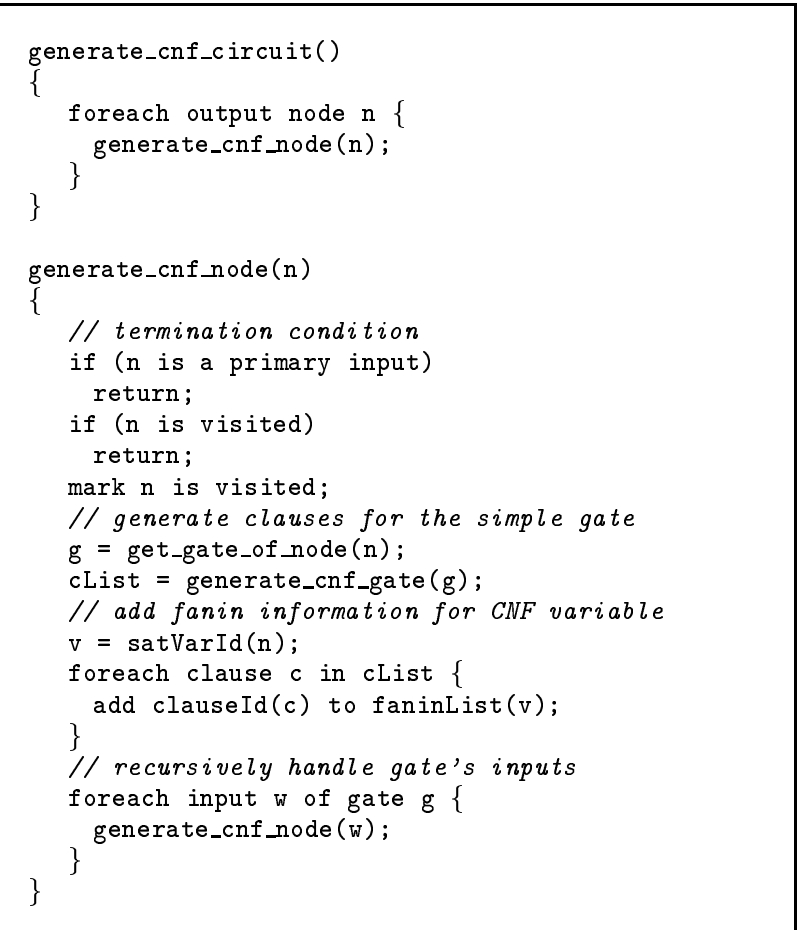

Figure 2: Pseudo-code for CNF Generation

We can easily handle complex combinational gates as well. A typical format for such gates is that of a table, where each row of the table identifies a tuple of allowable inputs and output(s), such as that used in VIS [3]. An example of CNF generation and structural information for such a table is shown in Figure 3. It also demonstrates how multivalued variables such as $x, y$, and equality constraints such as $(=y),(=z)$ are handled. Since complex gates usually result in introduction of many extra $\mathrm{CNF}$ variables, we use a post-processing phase of compacting the CNF description.

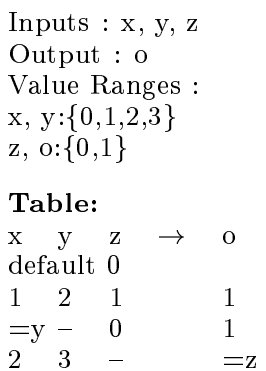

Each multi-valued variable is encoded by binary variables.

$\mathrm{x}: \mathrm{x} 0 \mathrm{x} 1$ (LSB)

$\mathrm{y}: \mathrm{y} 0 \mathrm{y} 1$ (LSB)

CNF Generation for Given Table:

A new variable is introduced for each row of table (v1,v2,v3). An equality constraint at the inputs also introduces new variables - one per Boolean variable $(\mathrm{v} 4, \mathrm{v} 5)$.

Clauses for introduced variables:

$v 1=\left(x 0^{\prime} \wedge x 1 \wedge y 0 \wedge y 1^{\prime} \wedge z\right)$

$v 2=\left(v 4 \wedge v 5 \wedge z^{\prime}\right)$

$v 3=\left(x 0 \wedge x 1^{\prime} \wedge y 0 \wedge y 1\right)$

$v 4=(x 0=y 0)$

$v 5=(x 1=y 1)$

Clauses for output per row:

$v 1 \rightarrow o$

$v 2 \rightarrow o$

$v 3 \rightarrow(o=z)$

(c8)

Negation of all row variables implies default value of output: $\left(v 1^{\prime} \wedge v 2^{\prime} \wedge v 3^{\prime}\right) \rightarrow o^{\prime} \quad(\mathrm{c} 9)$

Clauses are created for (c1)-(c9) using standard methods, without introducing any other variables.

$\begin{array}{ll}\text { Structural Information for Given Table } \\ \text { CNF Variable } & \text { Fanin List of Clauses } \\ \text { v1 } & \text { (c1) } \\ \text { v2 } & (\text { c2 }) \\ \text { v3 } & (c 3) \\ \text { v4 } & (c 4) \\ \text { v5 } & (c 5) \\ \text { o } & (c 6),(c 7),(c 8),(c 9)\end{array}$

Figure 3: Example for CNF Generation

\subsection{Detecting Inactive Clauses}

The pseudo-code for our procedure for detecting inactive clauses is shown in Figure 4. A recursive backward traversal is performed over all variables, starting from each variable denoting a circuit output. For each variable, we loop over the clauses in its fanin list, each of which is marked "active". If the clause is unsatisfied, then a recursive traversal is invoked for each of the variables appearing in the clause. This is because each of these variables, whether assigned or unassigned, is "observable" in the sense that it can affect the satisfiability status of the clause. On the other hand, if the clause is satisfied, then only its assigned variables are "observable". Indeed, each unassigned variable of a satisfied clause is "unobservable" in the sense that it cannot affect its satisfiability status. Therefore, we do not invoke backward traversal for such variables. Our marking procedure is safe, i.e. any clause which is unmarked at the end is guaranteed to be inactive. (The simple connectivity information we use 


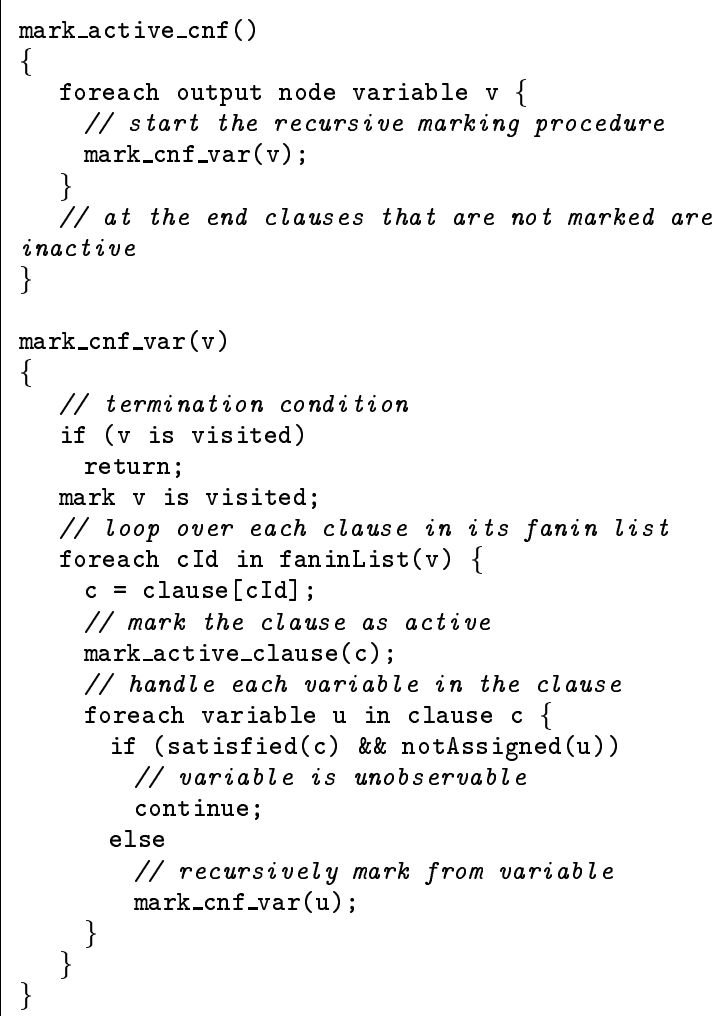

Figure 4: Pseudo-code for CNF Marking

is not enough to make an exact determination of all unobservable gates. For simple gates, the additional information required is identification of controlling input values, but this is hard to generalize for complex combinational gates described earlier.)

In terms of complexity, note that the traversal involves marking all clauses and variables in the worst case. Depending on the application, this cost can be either incurred at every decision level, or amortized over multiple decision levels within the SAT search. All clauses which are marked inactive need not be considered for satisfaction or for decision heuristics in the sub-tree rooted at that decision level. Note that these clauses may again become active after backtracking from this decision level.

\section{IMAGE COMPUTATION USING SAT}

Historically, symbolic state space traversal $[6,8]$ has relied on efficient algorithms based on BDDs [4] for carrying out an image computation, shown below:

$$
\operatorname{Image}(P, T)(y)=\exists_{x, i} T(x, i, y) \wedge P(x)
$$

Here, $x / y$ denote present/next state variables, $i$ denotes primary input variables, $\mathrm{T}$ denotes the transition relation, and $\mathrm{P}$ denotes the input state set. BDDs are used to represent the characteristic function of the transition relation, as well as the input/image sets. As an example application, the set of reachable states can be computed by starting from a set $P$ which denotes the set of initial states of a system, and using image computation iteratively, until a fixpoint is reached. The BDD-based approaches work well when it is possible to represent the sets of states and the transition relation (as a whole, or in a usefully partitioned form) using BDDs. Unfortunately, BDD size is very sensitive to the number of variables, variable ordering, and the nature of the logic expressions being represented.

Recently, an integration of SAT and BDDs has been proposed for image computation [10], which represents the transition relation as a CNF formula, and organizes the search for solutions as a top-level SAT search. BDD-based subproblems are invoked on-the-fly, in order to obtain multiple solutions simultaneously. The next section describes experimental results for reachability analysis based on this image computation engine.

\section{EXPERIMENTAL RESULTS}

There has been significant progress made in symbolic reachability analysis in recent years. We compare the SAT-based image computation with and without the inactive clause removal (ICR) optimization, to state of the art techniques in VIS [3], a public domain tool. Our prototype implementation of the SAT-based image computation algorithm uses the GRASP SAT solver [15] and the CUDD BDD package [21], and has been integrated within VIS. All reported experiments were run on an UltraSparc machine, with a $296 \mathrm{MHz}$ processor, and 1 GB memory. Dynamic variable reordering was enabled throughout. Unless noted otherwise, a time limit of 10 hours was used, and a "TT" in the results tables indicates a timeout, while an "SS" indicates a spaceout.

\subsection{Reachability Analysis}

Results for reachability analysis on the benchmark circuits are shown in Table 1. The name of the circuit appears in Column 1, and number of latches in Column 2 (marked \#L). The number of CNF variables and clauses are shown, respectively in Column 3 (marked \#V/\#C). Column 4 shows the number of image steps completed, and a "(C)" after the number of steps indicates that the traversal was complete, i.e. a fixpoint was reached. Column 5 shows the CPU time (in seconds) for standard VIS [3], which uses only BDDs for image computation. The remaining columns report the results for the SAT-based image computation method, which is labeled (VIS: SAT+BDDs) in the table. Columns 6 and 7 report the CPU time (in seconds) without and with ICR, respectively. Column 8 reports the speedup factor as a ratio of (time without ICR)/(time with ICR). Finally, Columns 9 and 10 report the percentage of clauses that were found inactive, and the percentage of clauses that were found inactive and unsatisfied. While the former number indicates the occurrence of inactive clauses, the latter number is a more useful figure of merit, since unsatisfied clauses critically affect SAT search in the sub-tree rooted at a decision level.

\subsection{Discussion}

Broadly speaking, the SAT-based image computation method does well in majority of the circuits when compared with standard VIS. It should be noted that our current contribution, i.e. the use of ICR, improves this method even further. In particular, the method with ICR performs better in 9 of the 10 benchmarks circuits compared to the SAT-based method without this optimization (Column 8). This clearly demonstrates that detecting inactive clauses and their re- 


\begin{tabular}{|c|c|c|c|c|c|c|c|c|c|}
\hline \multirow{3}{*}{$\begin{array}{l}\text { Circuit } \\
\text { Name }\end{array}$} & \multirow[t]{3}{*}{ 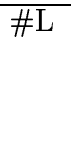 } & \multirow[t]{3}{*}{ \# $\mathrm{V} / \# \mathrm{C}$} & \multirow{3}{*}{$\begin{array}{l}\text { Image } \\
\text { Steps }\end{array}$} & \multirow{3}{*}{$\begin{array}{r}\text { VIS } \\
\text { (Standard) } \\
\text { Time }(\mathrm{s})\end{array}$} & \multicolumn{5}{|c|}{ SAT-based Method (VIS: SAT+BDDs) } \\
\hline & & & & & No ICR & & & $\overline{\text { With ICR }}$ & \\
\hline & & & & & Time(s) & Time(s) & $\begin{array}{l}\text { Speedup } \\
\text { Ratio }\end{array}$ & $\begin{array}{r}\% \text { Inactive } \\
\text { Clauses }\end{array}$ & $\begin{array}{l}\text { \% Inactive and } \\
\text { Unsat Clauses }\end{array}$ \\
\hline$\overline{\mathrm{s} 1269}$ & $\overline{37}$ & 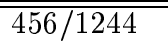 & 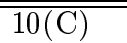 & 3269 & 2688 & $\overline{2164}$ & $\overline{1.24}$ & "60.9 & $\overline{14.9}$ \\
\hline$\overline{\mathrm{s} 1512}$ & 57 & $496 / 1301$ & $1024(\mathrm{C})$ & 2647 & 5753 & 5768 & 1.00 & 18.7 & 0.9 \\
\hline s3271 & 116 & $1183 / 3219$ & $17(\mathrm{C})$ & 17933 & 14793 & 14036 & 1.05 & 48.7 & 28.6 \\
\hline s3330 & 132 & $846 / 2114$ & $9(\mathrm{C})$ & 20029 & 3967 & 2029 & 1.96 & 41.2 & 15.7 \\
\hline prolog & 136 & $1027 / 2607$ & $\begin{array}{l}4 \\
9(\mathrm{C})\end{array}$ & $\begin{array}{r}25003 \\
\text { TT }\end{array}$ & $\begin{array}{r}765 \\
11175\end{array}$ & $\begin{array}{r}674 \\
4697\end{array}$ & 2.38 & 36.8 & 12.8 \\
\hline s5378 & 164 & $1012 / 2819$ & $\begin{array}{l}8 \\
45(\mathrm{C})\end{array}$ & $\begin{array}{r}57986 \\
\text { SS }>30 h\end{array}$ & $\begin{array}{r}5957 \\
60500\end{array}$ & $\begin{array}{r}6016 \\
90337\end{array}$ & 0.67 & 28.3 & 5.3 \\
\hline s1423 & 74 & $574 / 1464$ & $\begin{array}{l}11 \\
12 \\
13\end{array}$ & $\begin{array}{r}8791 \\
\text { TT } \\
\text { TT }\end{array}$ & $\begin{array}{r}3010 \\
9577 \\
\text { TT }\end{array}$ & $\begin{array}{r}3318 \\
5423 \\
13151\end{array}$ & 1.77 & 32.7 & 4.7 \\
\hline s3384 & 183 & $1187 / 2853$ & $\begin{array}{l}4 \\
5 \\
6\end{array}$ & $\begin{array}{r}24875 \\
\text { TT } \\
\text { TT }\end{array}$ & $\begin{array}{r}787 \\
2882 \\
\text { TT }\end{array}$ & $\begin{array}{r}557 \\
1095 \\
7801\end{array}$ & 2.63 & 24.0 & 0 \\
\hline $\mathrm{s} 9234.1$ & 211 & $2316 / 6548$ & $\begin{array}{l}7 \\
9\end{array}$ & $\begin{array}{r}2360 \\
11577\end{array}$ & $\begin{array}{r}8030 \\
\text { TT }\end{array}$ & $\begin{array}{r}4569 \\
22777\end{array}$ & 1.76 & 31.1 & 9.3 \\
\hline s13207.1 & 638 & $3464 / 8773$ & $\begin{array}{l}8 \\
10 \\
14\end{array}$ & $\begin{array}{r}2285 \\
4906 \\
28600 \\
\end{array}$ & $\begin{array}{r}28025 \\
\text { TT } \\
\text { TT }\end{array}$ & $\begin{array}{r}5061 \\
8340 \\
\text { TT }\end{array}$ & 5.54 & 29.9 & 5.6 \\
\hline
\end{tabular}

Table 1: Reachability Results for Benchmark Circuits

moval is beneficial to the underlying search. Not surprisingly, the benefit seems to be greater when more inactive clauses are found, as indicated by a higher number in Column 9. Though not shown in the table, the overhead of applying ICR is minimal, constituting less than 10 seconds of CPU time in the largest experiment.

In terms of details, the SAT-based methods performs fairly well for the first 4 "easy" circuits where all methods complete traversal. In particular, for s3330, the method with ICR outperforms standard VIS by an order of magnitude. Recently, other improvements over standard VIS have also been reported for these circuits [16, 17]. However, since these implementations are not publicly available, we were unable to conduct comparison experiments within our environment.

The real benefit of SAT-based image computation is demonstrated by the next two circuits - prolog, and s5378. The SAT-based method is successful in performing a complete traversal, both with and without ICR, whereas VIS is not. For these circuits, and the next two circuits - s1423, and s3384 - the SAT method is consistently faster, sometimes by two orders of magnitude, and completes more steps through use of ICR. Finally, the last two circuits - s9234, and s13207 - have a bigger CNF representation than the other circuits, making the SAT-based method less effective. For these two circuits, the use of ICR improves the SAT-based method by greater than factors of 2 , and 5 , respectively. This has the benefit of closing the performance gap between the SATbased method and standard VIS.

In the SAT-based image computation application, ICR affects both the decision making in SAT, as well as size of the BDD sub-problems which is directly related to the number of unsatisfied clauses. The result in Column 10 for circuit s3384 is very interesting for this reason. Since there are no inactive unsatisfied clauses, the size of the BDD sub- problems does not change. However, it is due to the different decision making in SAT (leading perhaps to different BDD sub-problems) that we get a speedup factor of 2.63. To demonstrate this effect of ICR, we present details of the experimental results for the prolog circuit in Table 2, where labels of the Columns are similar to Table 1. Note that use of ICR results in less number of BDD leaves (sub-problems) in most image computation steps of the complete traversal. Also, the fraction of inactive clauses and inactive unsatisfied clauses remains consistently high in all steps. More tellingly, the overall peak BDD nodes and the maximum live BDD nodes (as reported by the CUDD package) are considerably lower with the use of ICR.

\section{CONCLUSIONS}

We have proposed an optimization technique which incorporates circuit-specific knowledge in order to improve performance of SAT algorithms on problems arising in VLSI CAD. This optimization is based on detection and removal of inactive clauses in the SAT formula, thereby reducing the size of the sub-problems. We also provide efficient algorithms for implementing this optimization within a $\mathrm{CNF}$ framework. We highlighted the impact of this optimization on a recently proposed application - SAT-based image computation for reachability analysis. We presented practical results for a number of benchmark circuits, which show a consistent performance improvement due to our optimization. We believe that more domain-specific optimizations of this nature are possible for SAT.

\section{REFERENCES}

[1] M. Abramovici, M. A. Breuer, and A. D. Friedman. Digital Systems Testing and Testable Design. Electrical Engineering, Communications and Signal 


\begin{tabular}{|c|c|c|c|c|c|c|c|}
\hline \multicolumn{2}{|c|}{ prolog circuit } & \multicolumn{2}{|c|}{$\overline{\text { No ICR }}$} & \multicolumn{4}{|c|}{$\overline{\text { With ICR }}$} \\
\hline Step & Reached States & $\begin{array}{r}\text { Time } \\
(\mathrm{s})\end{array}$ & $\begin{array}{r}\text { \# BDD } \\
\text { Leaves }\end{array}$ & $\begin{array}{r}\text { Time } \\
(\mathrm{s})\end{array}$ & $\begin{array}{r}\text { \# BDD } \\
\text { Leaves }\end{array}$ & $\begin{array}{r}\% \text { Inactive } \\
\text { Clauses }\end{array}$ & $\begin{array}{l}\text { \% Inactive and } \\
\text { Unsat Clauses }\end{array}$ \\
\hline$\overline{11}$ & $3.77487 \mathrm{e}+07$ & $\overline{18.1}$ & 2 & $\overline{17.2}$ & 2 & 31.8 & $\overline{14.5}$ \\
\hline 2 & $4.93249 \mathrm{e}+09$ & 7.4 & 3 & 7.1 & 3 & 32.9 & 14.3 \\
\hline 3 & $5.95738 \mathrm{e}+14$ & 35.1 & 11 & 55.2 & 12 & 38.8 & 16.0 \\
\hline 4 & $1.29149 \mathrm{e}+16$ & 705.2 & 24 & 595.0 & 22 & 36.9 & 12.4 \\
\hline 5 & $1.7276 \mathrm{e}+17$ & 1872.9 & 24 & 2671.3 & 22 & 36.9 & 12.4 \\
\hline 6 & $5.99378 \mathrm{e}+17$ & 2814.7 & 26 & 645.8 & 22 & 36.9 & 12.4 \\
\hline 7 & $7.22992 \mathrm{e}+17$ & 2108.5 & 26 & 380.2 & 22 & 36.9 & 12.4 \\
\hline 8 & $7.2778 \mathrm{e}+17$ & 3485.1 & 40 & 293.6 & 22 & 36.9 & 12.4 \\
\hline 9 & $7.2778 \mathrm{e}+17$ & 128.1 & 23 & 32.0 & 22 & 36.9 & 12.4 \\
\hline \multirow[t]{2}{*}{ Total } & $7.2778 \mathrm{e}+17$ & 11175.0 & 179 & 4697.5 & 149 & 36.8 & 12.8 \\
\hline & & \multicolumn{2}{|c|}{$\begin{array}{l}\text { Peak BDDs }=8.7 \mathrm{M} \\
\text { Live BDDs }=2.7 \mathrm{M}\end{array}$} & \multicolumn{4}{|c|}{$\begin{array}{l}\text { Peak BDDs }=2.7 \mathrm{M} \\
\text { Live BDDs }=0.9 \mathrm{M}\end{array}$} \\
\hline
\end{tabular}

Table 2: Details of Results for prolog circuit

Processing. Computer Science Press, New York, New York, 1990.

[2] A. Biere, A. Cimatti, E. M. Clarke, and Y. Zhu. Symbolic model checking without BDDs. In Tools and Algorithms for the Analysis and Construction of Systems (TACAS), volume 1579 of Lecture Notes in Computer Science, 1999.

[3] R. K. Brayton et al. VIS: A system for verification and synthesis. In R. Alur and T. Henzinger, editors, Proceedings of the Internation Conference on Computer-Aided Verification, volume 1102 of Lecture Notes in Computer Science, pages 428-432, June 1996.

[4] R. E. Bryant. Graph-based algorithms for Boolean function manipulation. IEEE Transactions on Computers, C-35(8):677-691, Aug. 1986.

[5] J. Burch and V. Singhal. Tight integration of combinational verification methods. In Proceedings of the International Conference on Computer-Aided Design, pages 570-576, 1998.

[6] J. R. Burch, E. M. Clarke, D. E. Long, K. L. McMillan, and D. L. Dill. Symbolic model checking for sequential circuit verification. IEEE Transactions on Computer-Aided Design of Integrated Circuits and Systems, 13(4):401-424, Apr. 1994.

[7] S. T. Chakradhar, V. D. Agrawal, and S. G. Rothweiler. A transitive closure algorithm for test generation. IEEE Transactions on Computer-Aided Design of Integrated Circuits and Systems, 12(1):1015-1028, July 1993.

[8] O. Coudert, J. C. Madre, and C. Berthet. Verifying temporal properties of sequential machines without building their state diagrams. In Proceedings of the Internation Conference on Computer-Aided Verification, volume 3 of DIMACS Series in Discrete Mathematics and Theoretical Computer Science, 1991.

[9] A. Gupta and P. Ashar. Integrating a Boolean satisfiability checker and BDDs for combinational verification. In Proceedings of the VLSI Design Conference, Jan. 1998.

[10] A. Gupta, Z. Yang, A. Gupta, and P. Ashar. Sat-based image computation with application in reachability analysis. In Proceedings of the Conference on Formal Methods in Computer-Aided Design, 2000.
[11] W. Kunz and D. Pradhan. Recursive learning: A new implication technique for efficient solutions to CAD problems - test, verification and optimization. IEEE Transactions on Computer-Aided Design of Integrated Circuits and Systems, 13(9):1143-1158, Sep. 1994.

[12] T. Larrabee. Test pattern generation using Boolean satisfiability. IEEE Transactions on Computer-Aided Design of Integrated Circuits and Systems, 11(1):4-15, Jan. 1992.

[13] J. P. Marques-Silva and A. L. Oliveira. Improving satisfiability algorithms with dominance and partitioning. In IEEE/ACM International Workshop on Logic Synthesis, May 1997.

[14] J. P. Marques-Silva and K. A. Sakallah. Grasp: A new search algorithm for satisfiability. In Proceedings of the International Conference on Computer-Aided Design, pages 220-227, Nov. 1996.

[15] J. P. Marquez-Silva. Grasp package. http://algos.inesc.pt/ jpms/software.html.

[16] I.-H. Moon, G. Hachtel, and F. Somenzi. Border-block triangular form and conjunction schedule in image computation. In Proceedings of the Conference on Formal Methods in Computer-Aided Design, 2000.

[17] I.-H. Moon, J. Kukula, K. Ravi, and F. Somenzi. To split or to conjoin: The question in image computation. In Proceedings of the Design Automation Conference, pages 23-28, June 2000.

[18] M. Moskewicz, C. Madigan, Y. Zhao, L. Zhang, and S. Malik. Engineering a (super?) efficient SAT solver. In Proceedings of the Design Automation Conference, 2001.

[19] M. Sheeran and G. Stalmark. A tutorial on Stalmark's method of propositional proof. Formal Methods in System Design, 16(1), 2000.

[20] O. Shtrichman. Tuning SAT checkers for bounded model checking. In Proceedings of the Internation Conference on Computer-Aided Verification, 2000.

[21] F. Somenzi et al. CUDD: University of Colorado Decision Diagram Package. http://vlsi.colorado.edu/ fabio/CUDD/.

[22] H. Zhang. SATO: an efficient propositional prover. In International Conference on Automated Deduction, number 1249 in LNAI, pages 272-275, 1997. 